\title{
Aerodynamic characteristics of a free-flight scramjet vehicle in shock tunnel
}

\author{
Marie Tanno ${ }^{1}\left(\mathbb{D} \cdot\right.$ Hideyuki Tanno $^{2}$
}

Received: 31 December 2020 / Revised: 8 May 2021 / Accepted: 21 May 2021

(c) The Author(s) 2021

\begin{abstract}
A multi-component aerodynamic test for an airframe-engine integrated scramjet vehicle model was conducted in the freepiston shock tunnel HIEST. A free-flight force measurement technique was applied to the scramjet vehicle model named MoDKI. A new method using multiple piezoelectric accelerometers was developed based on overdetermined system analysis. Its unique features are the following: (1) The accelerometer's mounting location can be more flexible. (2) The measurement precision is predicted to be improved by increasing the number of accelerometers. (3) The angular acceleration can be obtained with single-axis translational accelerometers instead of gyroscopes. (4) Through the averaging process of the multiple accelerometers, model natural vibration is expected to be mitigated. With eight model-onboard single-axis accelerometers, the three-component aerodynamic coefficients (Drag, Lift, and Pitching moment) of MoDKI were successfully measured at the angle of attack from 0.7 to 3.4 degrees under a Mach 8 free-stream test flow condition. A linear regression fitting revealed a $95 \%$ prediction interval as the measurement precision of each aerodynamic coefficient.
\end{abstract}

\section{Graphical abstract}

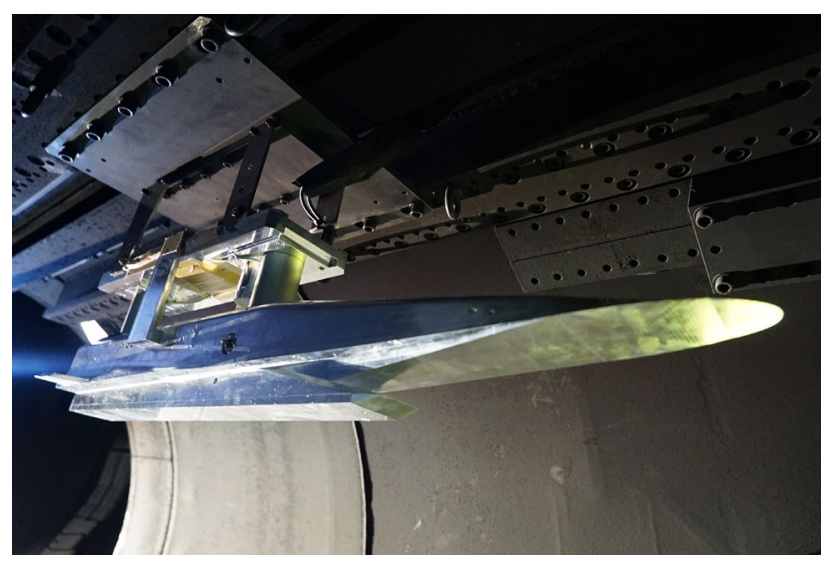

Marie Tanno

tanno.marie@jaxa.jp

1 Chofu Aerospace Center Aerodrome Branch, Japan Aerospace Exploration Agency, 6-13-1 Osawa, Mitaka-shi, Tokyo 181-0015, Japan

2 Department of Aerospace Engineering, Tohoku University, 6-6, Aramaki Aoba, Aoba-ku, Sendai, Miyagi 980-8579, Japan

\section{Introduction}

The scramjet engine is highly anticipated to be the most likely used propulsion system for the next-generation hypersonic transporter. Since the scramjet is impacted to such a considerable extent by the vehicle shape and configuration, one of the key technical issues is assessing the totally integrated vehicle, in terms of aerodynamic stability and propulsion performance. Although flight tests are one of the most desirable options for the integrated vehicle assessment, 
the testing cost is high, and the number of the test and flight conditions is both limited. Conversely, shock tunnels have been commonly used for hypersonic aerothermodynamic research at a reasonable operating cost. They can easily produce free-stream test flow over Mach 8 with the Reynolds number equivalent to actual flight condition, and many scramjet studies were conducted with such facilities (Stalker and Morgan 1982; Paull et al. 1995; Holden 2000; Itoh et al. 2002; Hannemann et al. 2014; Jiang et al. 2021). Since the facilities' test time is a few milliseconds, this precludes conventional force balances due to the excessive response time. Although the last couple of decades have seen many studies conducted using the force measurement technique, nevertheless it remains state-of-the-art. Most previous studies were limited to single-component (thrust) measurements, though several multi-component studies have been reported (Robinson et al. 2004, 2006; Doherty et al. 2015; Hannemann et al. 2015). Moreover, less attention was paid to the measurement precision, which is crucial to determine actual vehicle aerodynamic characteristics. For an integrated scramjet-powered vehicle, the longitudinal vehicle stability, namely pitching motion, should be assessed, including the measurement precision.

In the present study, a free-flight aerodynamic force measurement technique was applied to measure a scramjet vehicle's aerodynamic characteristics in the free-piston shock tunnel HIEST (Itoh et al. 2002), and a new analytical method using multiple single-axis accelerometers was developed. The three-component aerodynamic coefficients (Drag, Lift, and Pitching moment) of the 1.1-m long fully integrated scramjet vehicle MoDKI (Model of Demonstrator Kakuda Initiative) were successfully measured, and measurement precision is discussed.

\section{Free-flight technique in short test duration}

$m \frac{d^{2} x}{d t^{2}}+c \frac{d x}{d t}+k x=F$

A general aerodynamic force measurement system (Fig. 1) can be modeled as a one-dimensional forced vibration (Eq. (1)). In this equation, $F$ denotes the aerodynamic load, $m$ is the mass of the model, $c$ is the damping coefficient, and $k$ is the stiffness of the support device (e.g., sting). In conventional wind tunnels, mechanical vibration is damped by an extended test time, and the aerodynamic force can be measured as the elastic force $k x$. Conversely, in impulsive facilities, the mechanical vibration typically does not dampen within the short test duration, resulting in less accurate measurement. In this case, by reducing the stiffness $k$ as smaller as possible, the aerodynamic force can

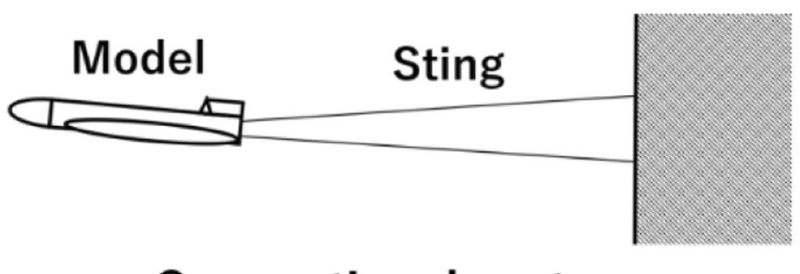

\section{Conventional system}

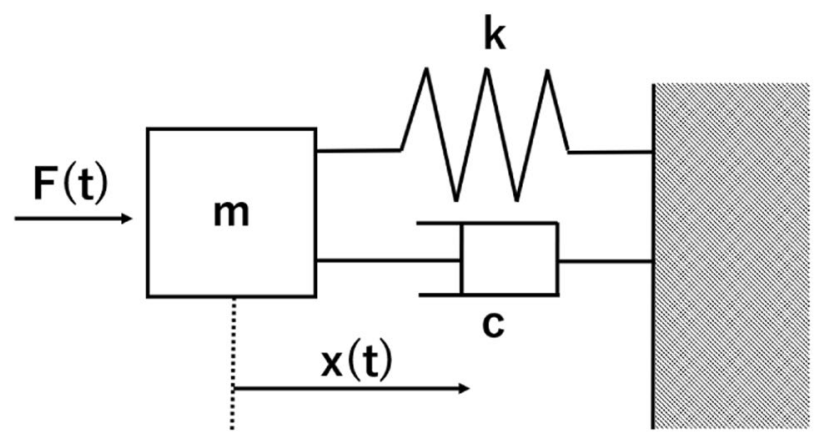

Fig. 1 Conventional aerodynamic measurement system and 1D vibration model

be measured as the inertial force $m\left(d^{2} x / d t^{2}\right)$. Based on the idea above, Duryea and Sheeran (1969) developed the socalled 'acceleration balance.' These balances have been used in several shock tunnel measurements (Reddy 1983; Joshi and Reddy 1986). In this technique, the models were weakly constrained by wires, rubbers, or springs instead of a rigid support system. The aerodynamic load can be obtained from the products of the measured acceleration and the mass of the model. However, due to the non-negligible drag produced by the weakly restrained support systems, sufficient measurement accuracy is not guaranteed, especially for multi-component force measurement (Tanno et al. 2009).

Free-flight force measurement techniques are one of the solutions for the issue. A good summary of previous researches can be found in Bernstein (1975). Most previous works were based on optical measurements using a high-speed camera system. Displacements were obtained directly from test-model images. Accelerations were then derived by double differentiation of displacement curves. However, since the measurement accuracy directly depends on the model displacement resolution, it will be naturally more challenging to keep the resolution as the test time becomes short. Instead of optical tracking, Naumann et al. (1993) used miniature accelerometers mounted inside the model in his shock tunnel measurement. Nevertheless, the model injection system and umbilicals may have produced aerodynamic interference, and such interference would become mostly undesirable when pitching moment measurements are required. 
A new free-flight technique was developed to keep the resolution and avoid the interferences using specially designed model-onboard data recording systems (Tanno et al. 2014). Since the test model is in free-fall during the test duration resulting in an entirely unconstrained motion, aerodynamic interference and mechanical vibration are eliminated. In his study, a pair of translational accelerometers was adopted to measure rotational motions instead of gyroscopes. A gyroscope is a favorable option to measure non-restrained or weakly restrained objects (Padgaonkar et al. 1975). However, the general gyroscope's frequency response, namely up to a few hundred $\mathrm{Hz}$, is insufficient for short-duration impulsive facilities such as HIEST. Instead, a translational accelerometer represents a reasonable option due to its high-frequency response $(>10 \mathrm{kHz})$, low cost, and availability. Theoretically, rotational motion can be identified with a pair of translational accelerometers offset from the rotation center. However, there is sensor-to-sensor variability on the accelerometers and errors associated with mounting location, just a single (for translation) or a pair (for rotation) of accelerometers may not suffice to improve precision. Moreover, when mounted, accelerometers may not always be optimally positioned given the general limitations on models in space. In fully integrated scramjet vehicle models, the available space may be limited due to the onboard fuel and electrical systems such as gas-fuel bottles, fuel-valves, solid-state timers, and batteries. These constraints make the design of the model challenging and could significantly impair the quality of the measurements. The following section introduces a new analytical approach applied to deal with the issues above.

\section{Analysis method for aerodynamic coefficient}

\subsection{Equation for the rotational motion of a rigid body}

Acceleration $\boldsymbol{A}_{\boldsymbol{P}}(t)$ at an arbitrary point, $\boldsymbol{P}$, within a rigid body is expressed by the following equation Eq. (2) with the distance from the center of rotation, $\boldsymbol{r}$, the translational acceleration $\boldsymbol{A}_{0}(t)$, the centrifugal acceleration $\boldsymbol{\omega}(t) \times \boldsymbol{\omega}(t) \times \boldsymbol{r}$, the angular acceleration $\dot{\boldsymbol{\omega}}(\boldsymbol{t}) \times \boldsymbol{r}$, and the gravitational acceleration $\boldsymbol{g}$. The coordinate system includes the accelerations around the y-axis, as shown in Fig. 2.

$A_{P}(t)=A_{0}(t)+\omega(t) \times \omega(t) \times \boldsymbol{r}+\dot{\omega}(t) \times \boldsymbol{r}+\boldsymbol{g}$

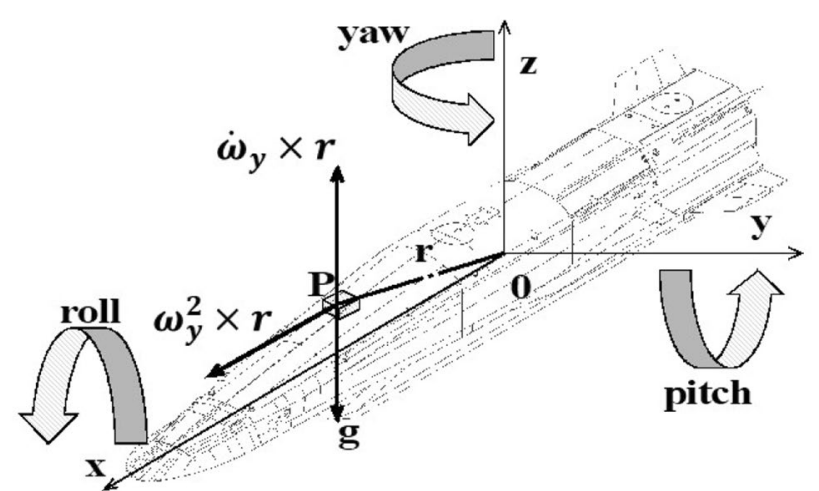

Fig. 2 Coordinate system of a measuring point. Centrifugal and angular acceleration were indicated around the $y$-axis

Given the very limited test time (in the order of a few milliseconds) and a model weight as heavy as several dozen kilograms, the angular velocity can be assumed to be small $(\omega(t) \approx 0)$.

Thus, the centrifugal acceleration term should be

$\omega(t) \times \omega(t) \times \boldsymbol{r} \approx 0$

Since the variation in model attitude was ignorable $(\omega(t) \approx 0)$, the gravitational acceleration $\boldsymbol{g}$ can be regarded as constant during the test period. Besides, the accelerometers are of differential type (AC-response accelerometer such as a piezoelectric type), making it possible to ignore the gravitational effects. From the above, Eq. (2) can be hence rewritten as follows.

$A_{P}(t)=A_{0}(t)+\dot{\omega}(t) \times r$

From Eq. (4), only translational and angular accelerations are included in the measured acceleration values.

\subsection{Calculation of multi-component force for the overdetermined system}

As mentioned in Sect. 2, two accelerometers per axis are theoretically required to measure moments with single-axis accelerometers. Since translational force can be measured simultaneously with moments, at least six accelerometers are necessary to determine the six-degree of freedom motion. However, actual instrumentation generally requires more accelerometers because of the issues described in Sect. 2 . Consequently, the measurement system becomes an overdetermined system, which is solved using linear regression theory.

For a measurement system with $N$ accelerometers, Eq. (4) can be expressed as the following matrix from Eq. (5). 


$$
\begin{aligned}
& \{A\}=[R]\{v\} \\
& \{A\}=\left\{\begin{array}{c}
a_{1} \\
a_{2} \\
\vdots \\
a_{n}
\end{array}\right\} \\
& {[R]=\left\{\begin{array}{cccccc}
r_{x 1} & r_{y 1} & r_{z 1} & \delta_{x 1} & \delta_{y 1} & \delta_{z 1} \\
r_{x 2} & r_{y 2} & r_{z 2} & \delta_{x 2} & \delta_{y 2} & \delta_{z 2} \\
\vdots & \vdots & \vdots & \vdots & \vdots & \vdots \\
r_{x n} & r_{y n} & r_{z n} & \delta_{x n} & \delta_{y n} & \delta_{Z n} \\
& & & & &
\end{array}\right\},} \\
& \{v\}=\left\{\begin{array}{c}
\dot{\omega}_{x} \\
\dot{\omega}_{y} \\
\dot{\omega}_{z} \\
a_{x} \\
a_{y} \\
a_{z}
\end{array}\right\}
\end{aligned}
$$

The coefficient matrix $[R]$ expresses the values representing the position $\boldsymbol{r}$ and sensing direction $\delta$ of the accelerometers. Subscripts $x, y$, and $z$ represent the axial, normal, and side directions, respectively. For the six degrees of freedom, $\{v\}$ represents translational accelerations (axial, normal, and side) and angular acceleration (rolling, pitching, and yawing) of each axis at the center of rotation (the center of gravity) of the model. The sensing axes $\delta_{x}$, $\delta_{y}$, and $\delta_{z}$ are defined as follows.

$x$-direction $:\left\{\delta_{x n}, \delta_{y n}, \delta_{z n}\right\}=\{1,0,0\}$

$y$-direction $:\left\{\delta_{x n}, \delta_{y n}, \delta_{z n}\right\}=\{0,1,0\}$

$z$-direction $:\left\{\delta_{x n}, \delta_{y n}, \delta_{z n}\right\}=\{0,0,1\}$

For an arbitrary mounting direction, $\delta$ can be adjusted $-1 \leq \delta \leq 1$.

Solving Eq. (5), the three-component force and $\{v\}=\left\{\omega_{x} \omega_{y} \omega_{z} a_{x} a_{y} a_{z}\right\}^{T}$ can be obtained. Since the matrix equation is an overdetermined system, and $[R]$ is a singular matrix, no unique solution can be determined.

$[R]^{+}=\left([R]^{T}[R]\right)^{-1}[R]^{T}$

Introducing the pseudo-inverse matrix $[\mathrm{R}]^{+},\{v\}$ can be calculated as follows.

$\{v\}=[R]^{+}\{A\}$

Since this method is based on least square regression, the variance in the measurement corresponds to normal distribution. Accordingly, the variance, or the precision, is reduced theoretically with $1 / \sqrt{N-1}$ ( $N$ representing the number of accelerometers). However, some of the accelerometers were placed in an orthogonal direction, it should be noted that not all accelerometers have the scope to improve the variance.

This method has additional favorable features. The model's mechanical vibration may not be damped within the test time for models without sufficient stiffness, impacting measurement precision. This method is expected to mitigate such vibrations through the averaging process with several accelerometers. Besides, since the method allows flexible relocation of the accelerometers to arbitrary locations, vibration can be reduced by placing the accelerometers appropriately (such as at vibration node points). However, precise prediction via finite element computations or pretests is needed to determine the optimum location.

\section{Experimental setup}

\subsection{MoDKI model}

During the free-flight test, the 'Model of Demonstrator Kakuda Initiative (MoDKI),' a fully integrated scramjet vehicle around $1.1 \mathrm{~m}$ long, was used. The standout feature is a scramjet underneath the fuselage. All the fuel injection and electric instruments were included onboard the scramjet model, including a gas-fuel tank and line, a fast-acting valve, data recorders, timers, and batteries. The dimension and onboard instruments of MoDKI are shown in Fig. 3 and Table 1, respectively. The model is made of aluminum alloy/ YH75 (which resembles extra super duralumin A7075). This model is equipped with two magnetic stainless steel pads at the front and rear of the top and can be secured by electromagnets on the ceiling inside the shock tunnel's test section.

An inert gas (Helium gas) injection test was conducted, and the standard operation of the total injection system was confirmed. The gas injection test results are excluded from this article.

\subsection{Moment of inertia}

The moment of inertia of MoDKI was measured with the two-support wires' torsional pendulum.

As shown in Fig. 4, the MoDKI model is suspended by two parallel threads and oscillates the vertical axis when twisted. The moment of inertia $J$ can be calculated by the frequency $f$ of this vibration measured.

$J=\frac{m g a b}{4 \pi^{2} f^{2} l}$

While $m$ is the mass of the model, $a, b$ represents the distance between each suspension wire from the center of the mass, and $l$ is the length of the suspension wire. 


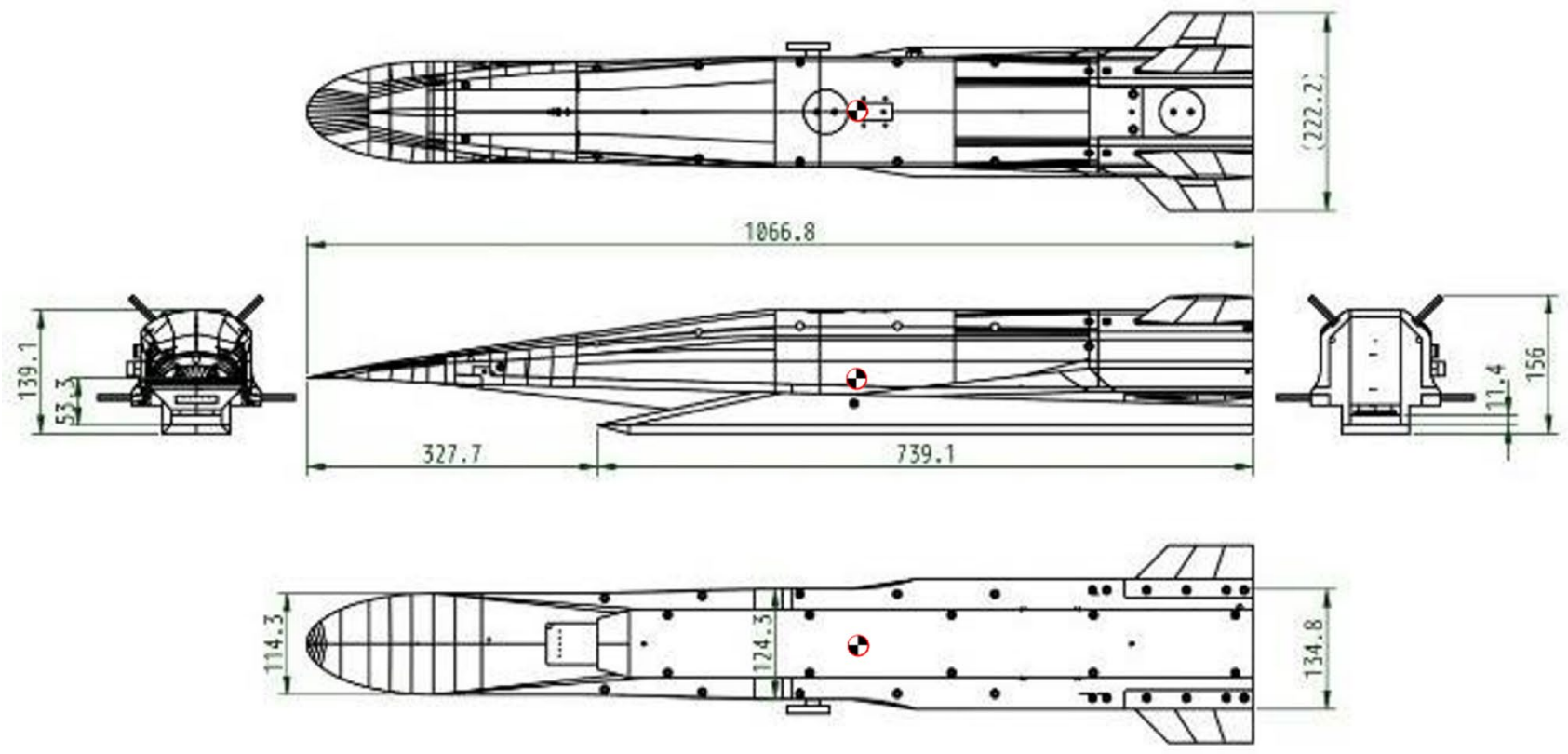

(a)

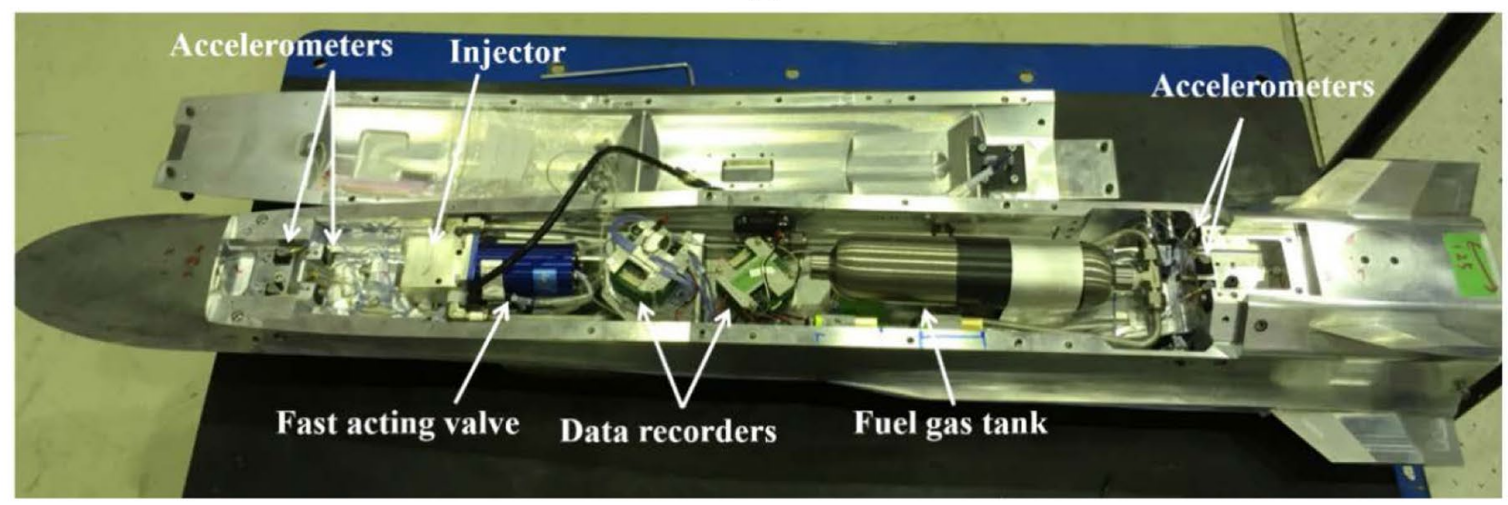

(b)

Fig. 3 a Drawings of the MoDKI and $\mathbf{b}$ onboard instruments

Table 1 MoDKI dimensions

\begin{tabular}{ll}
\hline Total length $[\mathrm{m}]$ & 1.1 \\
Total width $[\mathrm{m}]$ & 0.22 \\
Total mass $[\mathrm{kg}]$ & 18.96 \\
Center of gravity $X_{G}[\mathrm{~mm}]$ & $634.7 \pm 0.1$ \\
Center of gravity $Y_{G}[\mathrm{~mm}]$ & $0.40 \pm 0.3$ \\
Center of gravity $Z_{G}[\mathrm{~mm}]$ & $74.7 \pm 0.5$ \\
Moment of inertia $\left[\mathrm{kgm}^{2}\right]$ & $1.21 \pm 0.01$ \\
Projected frontal area $\left[\mathrm{m}^{2}\right]$ & 0.0112 \\
\hline
\end{tabular}

\subsection{Onboard sensors and data recorders}

For the surface pressure measurement, MoDKI has seven pressure transducers that were flush-mounted on the model surface. They were piezoresistive type XCQ093 or XCL-072 (Kulite Semiconductor Products Inc.), and their locations are shown in Fig. 5 and Table 2. For the force measurement, eight accelerometers were instrumented: the piezoelectric miniature single-axis accelerometer PCB352C65 (PCB Piezoelectronics, Inc.), which has a resonant frequency less than $35 \mathrm{kHz}$. Four of the eight accelerometers are arranged in the $\mathrm{X}$-axis direction and the remainder in the Z-axis direction. The positions and sensing axis of the test series accelerometers are shown in Fig. 5 and Table 3 (the sensing axis is shown in parentheses to the 


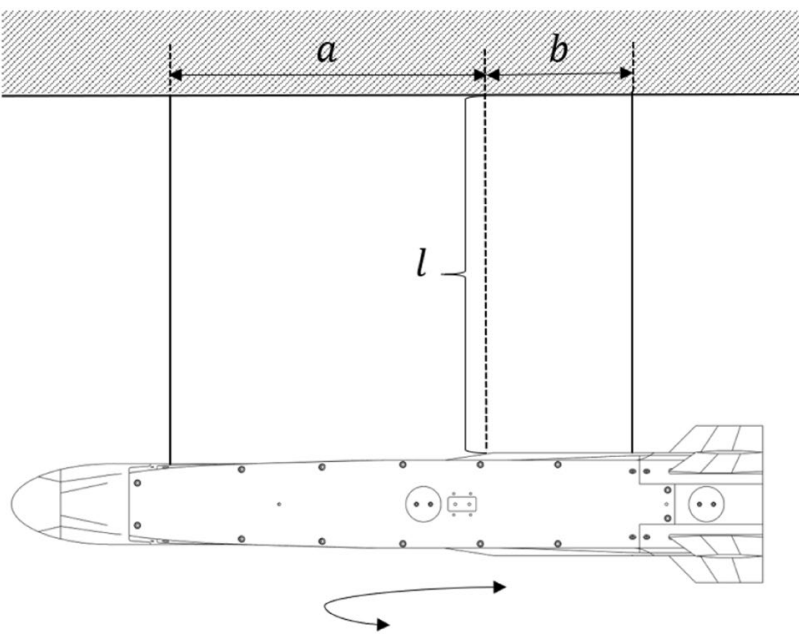

Fig. 4 Schematic of two-support wires vertical-axis torsional pendulum

right of each accelerometer number). The location uncertainty depends on the machining accuracy and is less than $0.05 \mathrm{~mm}$ in this model.
An onboard miniature data recorder is the critical technology for the present free-flight onboard measurements. In this study, two JAXA original data recorders (Tanno et al. 2014) were onboard in MoDKI, which records at a sampling rate of $500 \mathrm{kHz}$ with a 16-bit resolution. A total of 16 channels were used, 8 each for piezoelectric sensors (accelerometers) and piezoresistive sensors (pressure transducers). The recorder's total measurement time is $800 \mathrm{~ms}$, which sufficed to capture the entire test period.

\subsection{HIEST (High-Enthalpy Shock Tunnel)}

Figure 6 shows an overview of HIEST, a free-piston shock tunnel operated by JAXA Kakuda Space Center, which can be used to test aerodynamic models up to $50 \mathrm{~cm}$ long and scramjet models up to $3 \mathrm{~m}$ long. Due to the tuned operation (Itoh et al. 1998), the facility can produce high enthalpy and high Reynolds number flow with a comparatively long test time. Low-enthalpy conditions were used in the present study, equivalent to the flight conditions approximately at Mach 8. Figure 7 shows an example of pressure traces (stagnation pressure, free-stream Pitot pressure) and heat-flux

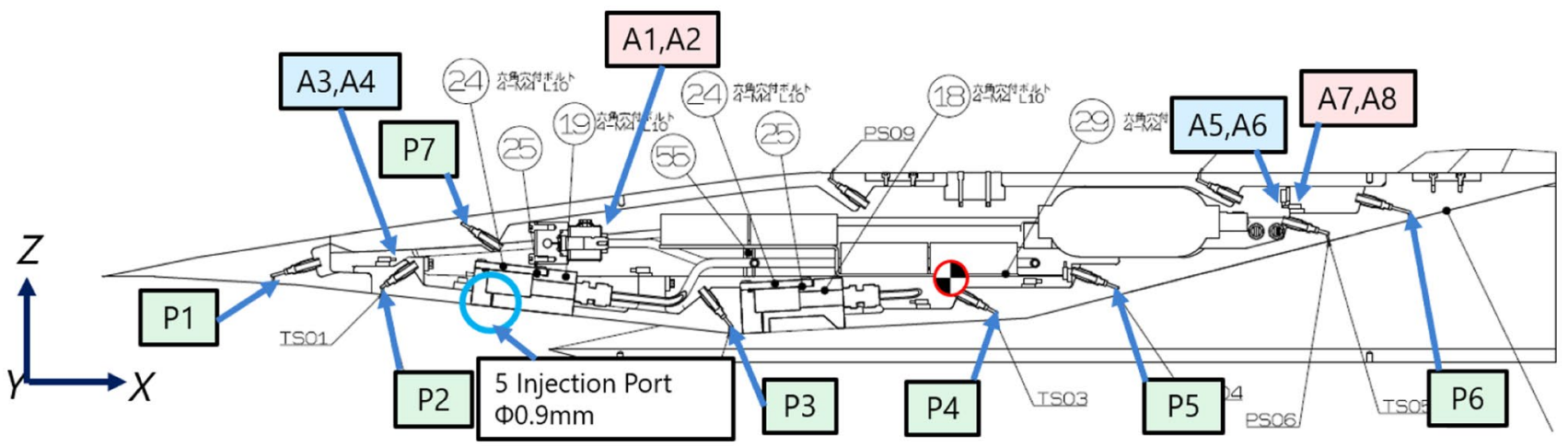

Fig. 5 Location of the accelerometers and pressure transducers mounted in MoDKI

Table 2 Pressure transducer location

Table 3 Accelerometer location

\begin{tabular}{llllllll}
\hline & P1 & P2 & P3 & P4 & P5 & P6 & P7 \\
\hline$X[\mathrm{~mm}]$ & 125.0 & 205.0 & 460.0 & 655.0 & 745.0 & 960.0 & 265.0 \\
$Y[\mathrm{~mm}]$ & 0.0 & 0.0 & 0.0 & 0.0 & 0.0 & 0.0 & 0.0 \\
$Z[\mathrm{~mm}]$ & 58.0 & 50.0 & 21.5 & 31.2 & 49.5 & 104.3 & 106.5 \\
\hline
\end{tabular}

\begin{tabular}{llllllll}
\hline & $\mathrm{A} 1(Z)$ & $\mathrm{A} 2(Z)$ & $\mathrm{A} 3(X)$ & $\mathrm{A} 4(X)$ & $\mathrm{A} 5(X)$ & $\mathrm{A} 6(X)$ & $\mathrm{A} 7(Z)$ \\
\hline$X[\mathrm{~mm}]$ & 438.0 & 438.0 & 224.8 & 224.8 & 862.8 & 862.8 & 876.8 \\
$Y[\mathrm{~mm}]$ & 28.5 & -28.5 & 15.0 & -15.0 & 25.0 & -25.0 & 10.0 \\
$Z[\mathrm{~mm}]$ & 71.9 & 71.9 & 72.6 & 72.6 & 114.1 & 114.1 & 118.1 \\
\hline
\end{tabular}




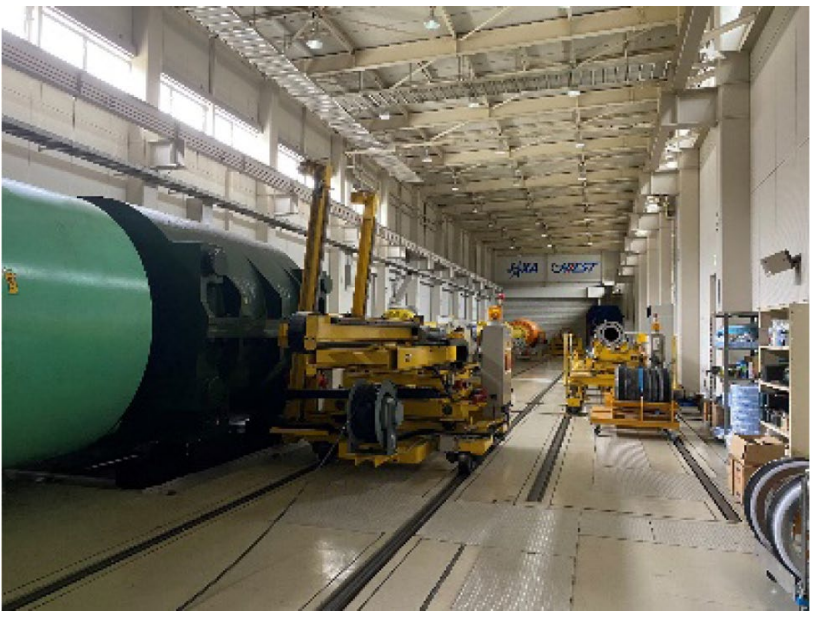

Fig. 6 Free-piston shock tunnel HIEST

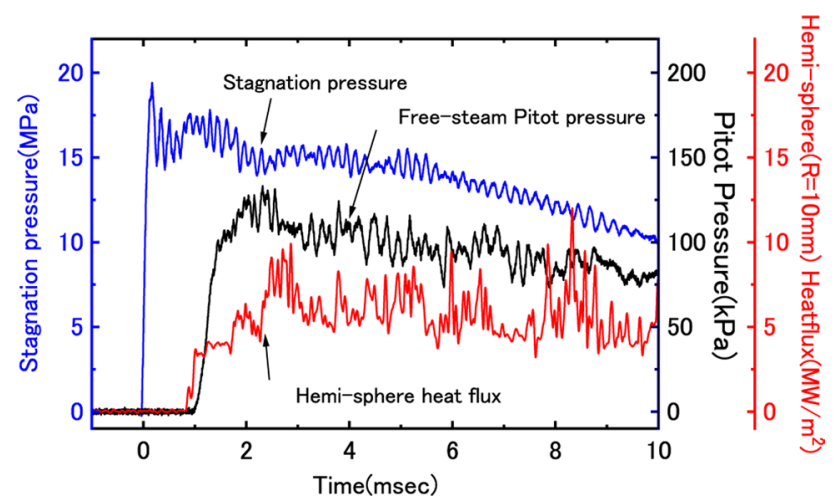

Fig. 7 Example of traces; stagnation pressure (shock tube end pressure), free-stream Pitot pressure, and a hemisphere (10 $\mathrm{mm}$ radius) probe heat-flux at the current condition

Table 4 HIEST test airflow conditions
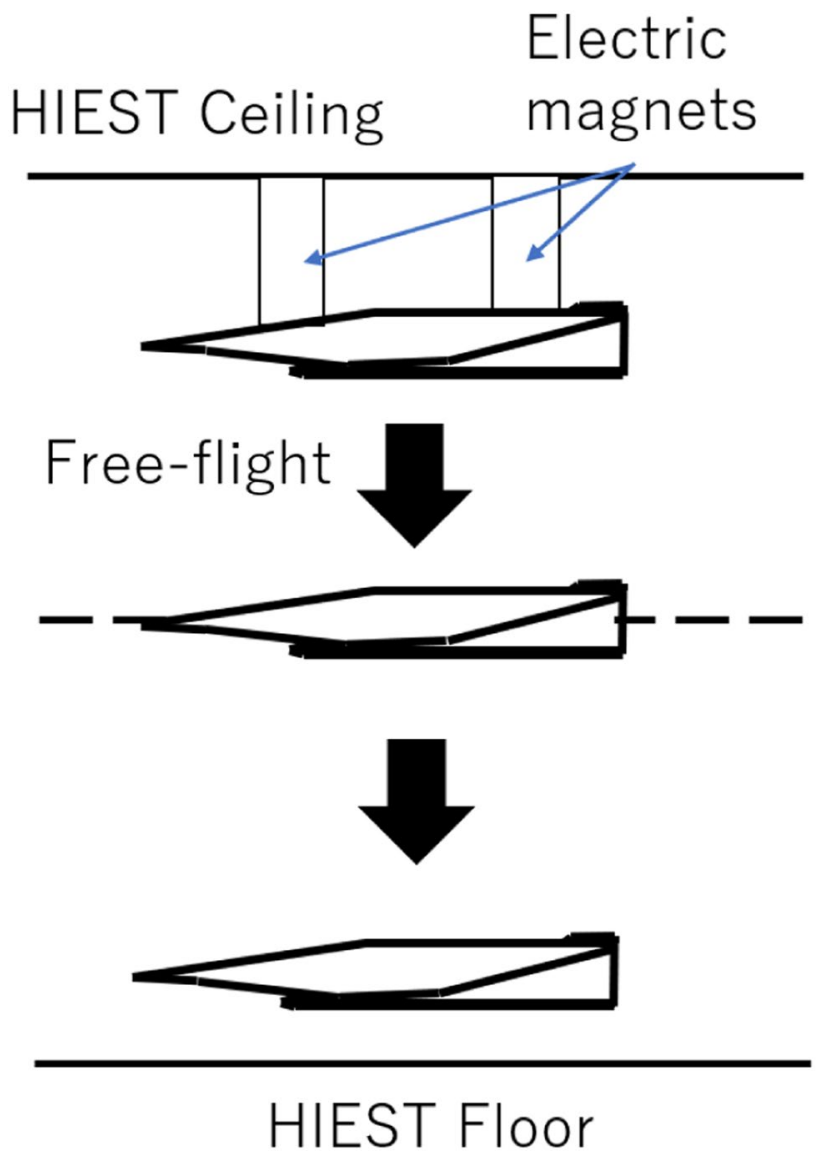

Fig. 8 Image of the free-flight procedure

measured with hemisphere probe (radius $10 \mathrm{~mm}$ ). HIEST test airflow conditions were calculated using the JAXA axisymmetric nozzle flow code (Takahashi et al. 2009), and the calculated free-stream parameters are shown in Table 4.

\subsection{Test procedure}

\begin{tabular}{lc}
\hline$p_{0}[\mathrm{MPa}]$ & $15.3 \pm 1.1$ \\
\hline$T_{0}\left[\times 10^{3} \mathrm{~K}\right]$ & $2.98 \pm 0.08$ \\
$H_{0}[\mathrm{MJ} / \mathrm{kg}]$ & $3.65 \pm 0.12$ \\
$p_{\infty}[\mathrm{kPa}]$ & $1.26 \pm 0.10$ \\
$T_{\infty}\left[\times 10^{2} \mathrm{~K}\right]$ & $2.36 \pm 0.08$ \\
$\rho_{\infty}\left[\times 10^{-2} \mathrm{~kg} / \mathrm{m}^{3}\right]$ & $1.58 \pm 0.09$ \\
$U_{\infty}[\mathrm{km} / \mathrm{s}]$ & $2.55 \pm 0.04$ \\
$\rho_{\infty} U_{\infty}^{2}\left[\times 10^{2} \mathrm{kPa}\right]$ & $1.03 \pm 0.07$ \\
$M_{\infty}$ & $7.64 \pm 0.04$ \\
$R e_{\infty}\left[\times 10^{6} 1 / \mathrm{m}\right]$ & $2.35 \pm 0.13$ \\
\hline
\end{tabular}

Figure 8 shows an image of the free-flight test procedure. In the initial status, MoDKI was held to the HIEST test section ceiling with two electromagnets. The model was released, deactivating the electromagnets $400 \mathrm{~ms}$ before the test flow arrival. The model release trigger was adjusted to position the free-falling model at the nozzle core flow during the test time. Since the onboard data recorder is pre-triggered, the pressure and acceleration histories were recorded throughout the entire test duration. Finally, the model drops onto the 


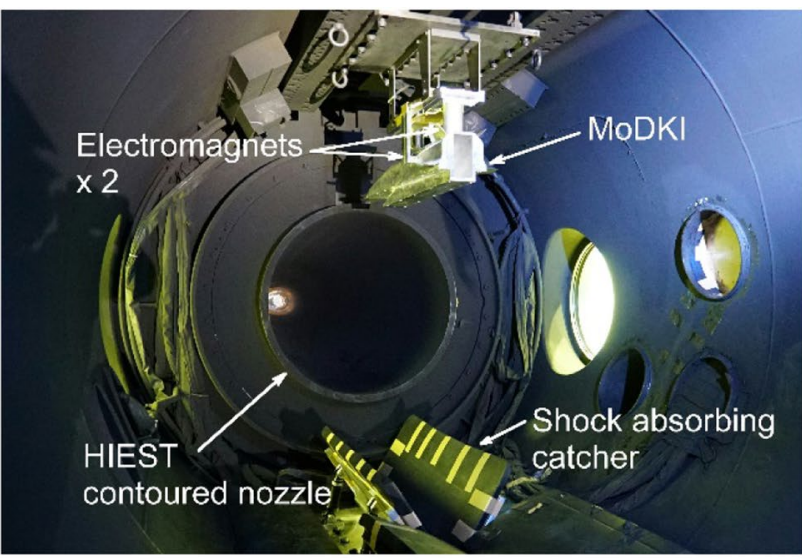

(a)

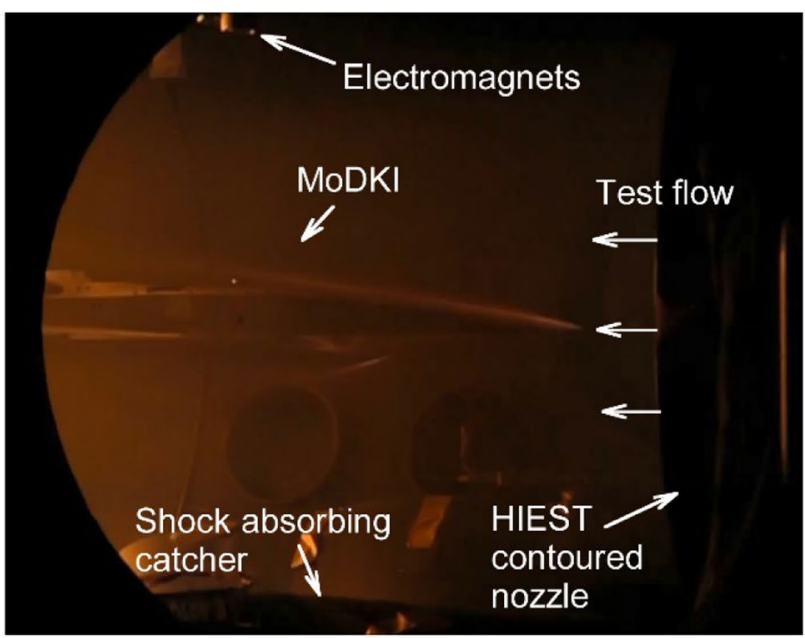

(b)

Fig. 9 a MoDKI installed on the HIEST test section ceiling. b Freeflying MoDKI during free flight

shock-absorbing catcher and is retrieved for data transfer from the data recorders to the host PC. Figure 9 shows a photograph of MoDKI installed on the HIEST test section ceiling and a high-speed camera image of MoDKI during free flight.

The exact angle of attack during the test time was monitored with an optical technique (Laurence 2012), which adopted Schlieren images taken with the high-speed camera (SHIMADZU Hypervision), and the model angle of attack

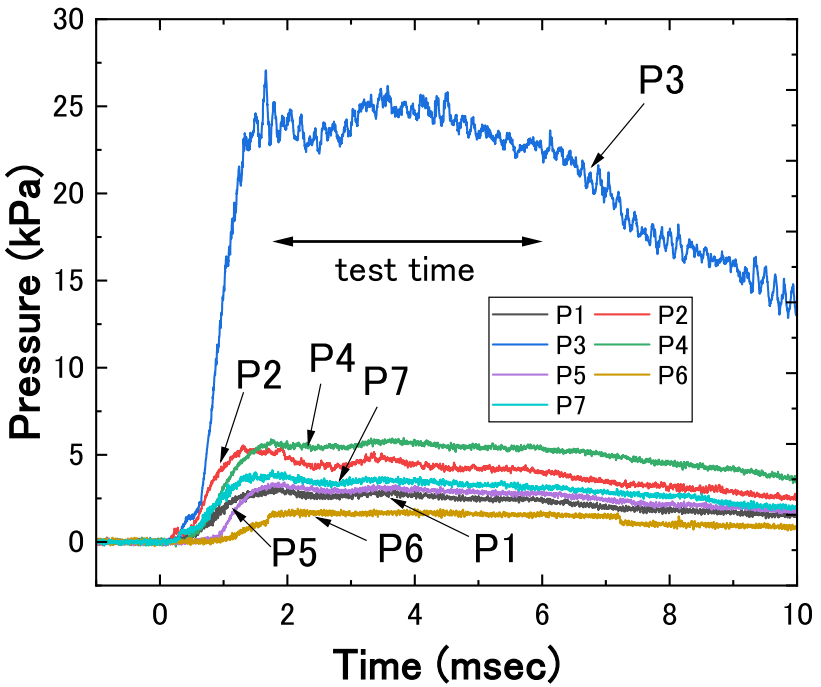

Fig. 10 Example of surface pressure histories

was measured for each shot. The AOA (angle of attack) varied from 0.7 to 3.4 degrees in the current test series. The test-to-test variation in measured AOA was \pm 0.5 from the targeted angle.

\section{Results and discussion}

\subsection{Pressure history}

Figure 10 shows an example of the model surface pressure traces. The surface pressure traces' starting process shows a gentle slope compared to the free-stream Pitot pressure trace (shown in Fig. 7) due to the flow establishment process of the model. The traces show virtually constant pressure from $\mathrm{t}=2$ to $6 \mathrm{~ms}$. The four milliseconds duration was hence defined as the test time.

\subsection{Aerodynamic characteristics of the MoDKI model}

As described in Sect. 4.3, the number of accelerometers was thus limited to eight due to the onboard data recorder's available channels. The measurement was limited to the three degrees of freedom (namely, three components), and components of the matrices are reduced as follows: 
Fig. 11 An example of the measured aerodynamic coefficients. a Drag coefficient, $\mathbf{b}$ Lift coefficient, and $\mathbf{c}$ Pitching moment coefficient

$$
\begin{aligned}
& \{A\}=[R]\{v\} \\
& \{A\}=\left\{\begin{array}{c}
a_{1} \\
a_{2} \\
a_{3} \\
a_{4} \\
a_{5} \\
a_{6} \\
a_{7} \\
a_{8}
\end{array}\right\}, \\
& {[R]=\left\{\begin{array}{ccc}
r_{1} & \delta_{x 1} & \delta_{z 1} \\
r_{2} & \delta_{x 2} & \delta_{z 2} \\
r_{3} & \delta_{x 3} & \delta_{z 3} \\
r_{4} & \delta_{x 4} & \delta_{z 4} \\
r_{5} & \delta_{x 5} & \delta_{z 5} \\
r_{6} & \delta_{x 6} & \delta_{z 6} \\
r_{7} & \delta_{x 7} & \delta_{z 7} \\
r_{8} & \delta_{x 8} & \delta_{z 8}
\end{array}\right\},} \\
& \{v\}=\left\{\begin{array}{c}
\dot{\omega}_{y} \\
a_{x} \\
a_{z}
\end{array}\right\}
\end{aligned}
$$

By applying the data reduction process above, bidirectional translational accelerations ( $\mathrm{X}$ and $\mathrm{Z}$-axes) and angular acceleration around the Y-axis (in pitch direction) were calculated.

$F_{D}=m\left(a_{x} \cos \theta+a_{z} \sin \theta\right)$

$F_{L}=m\left(a_{z} \cos \theta-a_{x} \sin \theta\right)$

$M_{y}=J \dot{\omega}_{y}$

From the products of mass and three accelerations calculated above, drag force $F_{D}$, lift force $F_{L}$, and pitching moment $M_{y}$ can be obtained. Since the current coordinate is the body axis system, the axial and normal force should be converted to drag and lift force as the above equations Eq. (11)-(13), where theta is the angle of attack and $J$ is the moment of inertia determined in Sect. 4.2.

$$
\begin{aligned}
& C_{D}=\frac{F_{D}}{\frac{1}{2} \rho_{\infty} U_{\infty}{ }^{2} S} \\
& C_{L}=\frac{F_{L}}{\frac{1}{2} \rho_{\infty} U_{\infty}{ }^{2} S}
\end{aligned}
$$

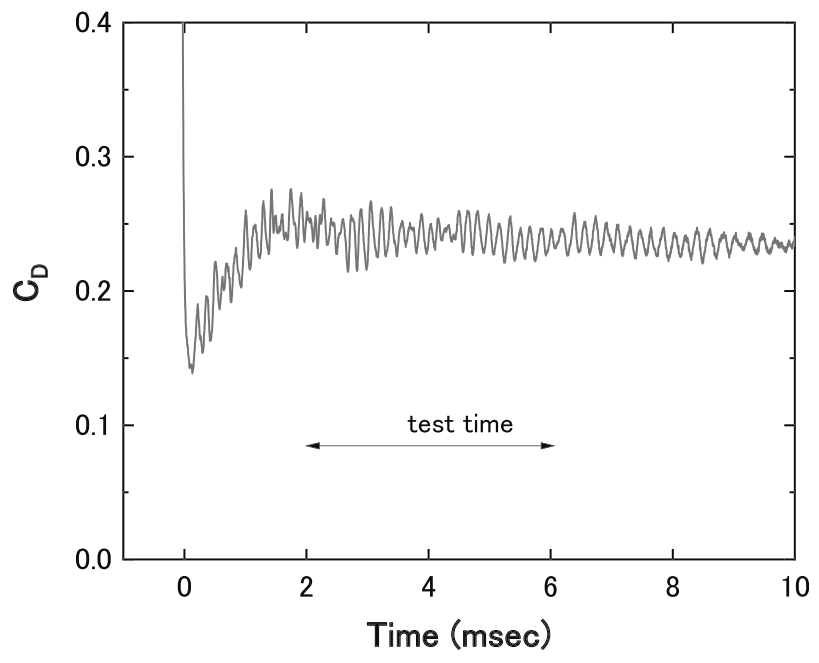

(a)

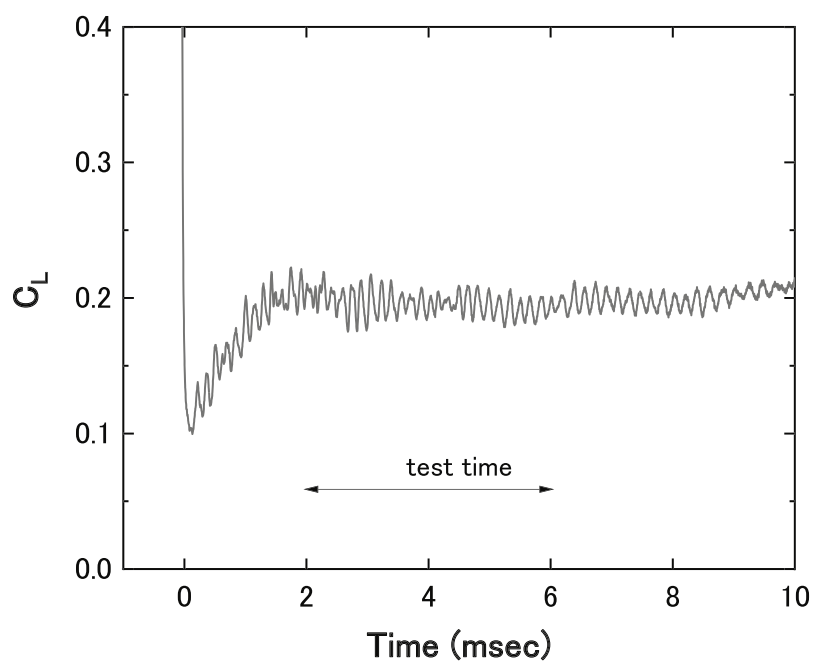

(b)

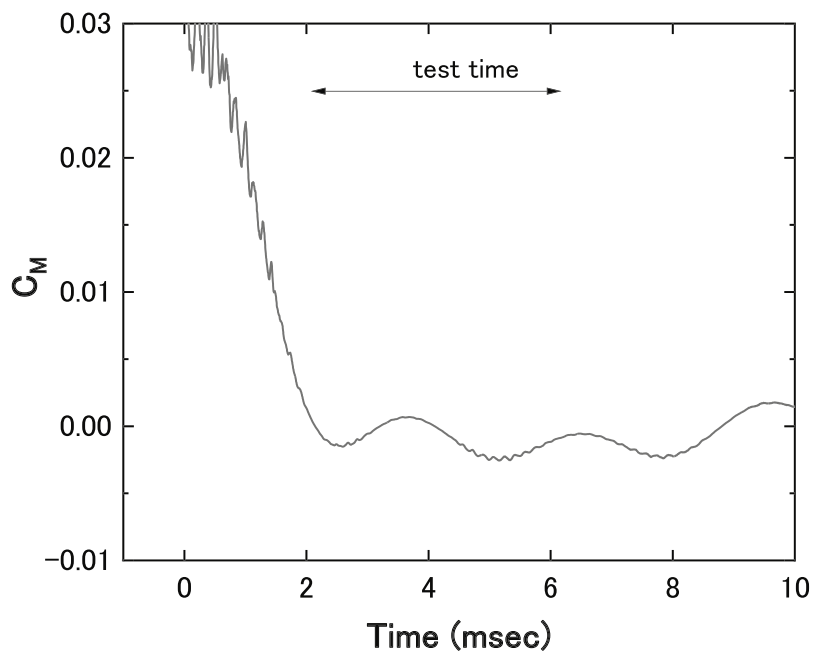

(c) 


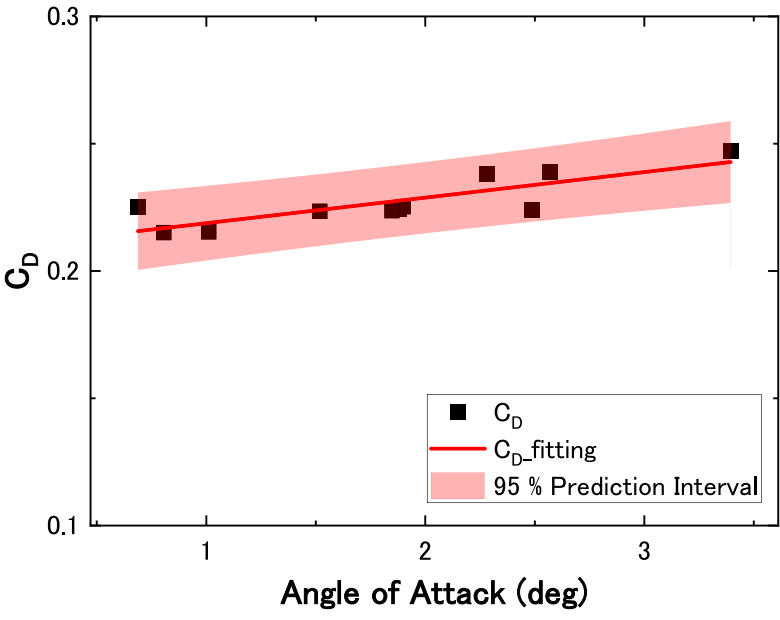

(a)

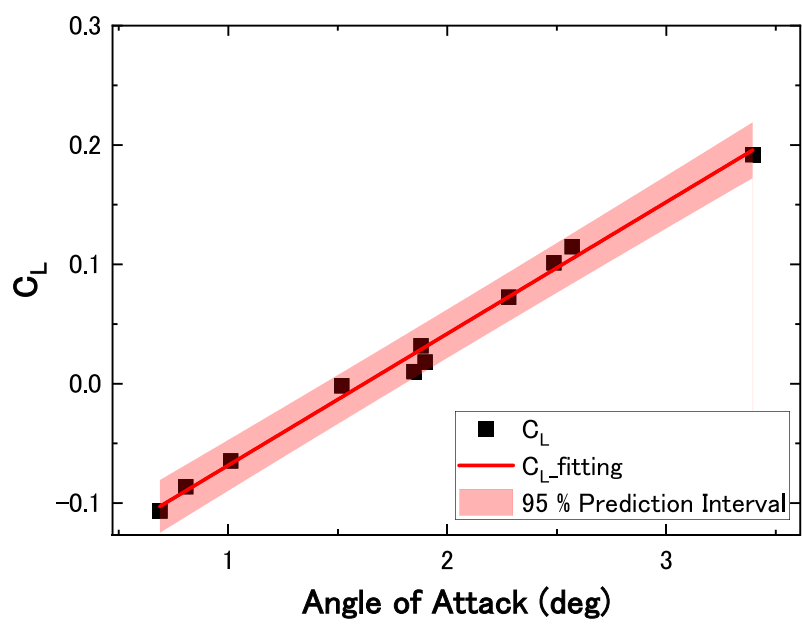

(b)

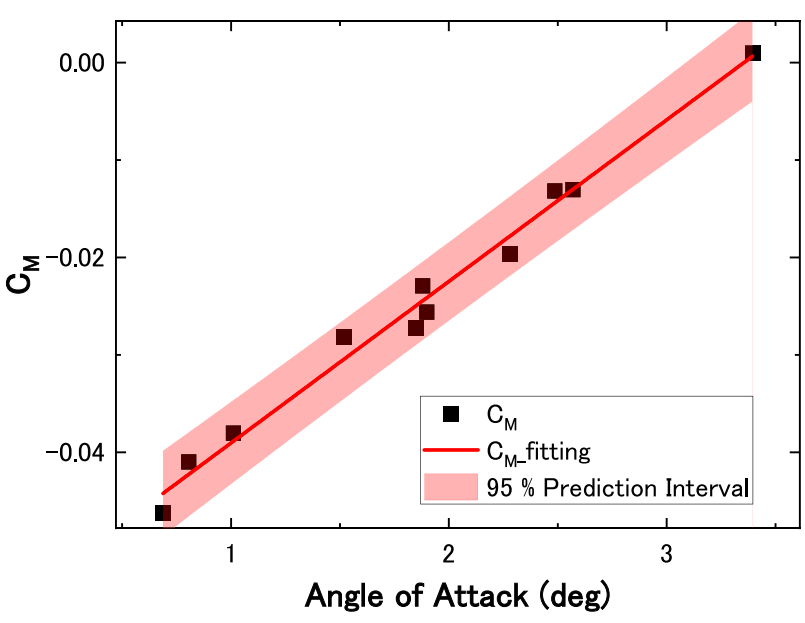

(c)

Fig. 12 The relationship between the model angle of attack and aerodynamic coefficient. a Drag coefficient, b Lift coefficient, and c Pitching moment coefficient

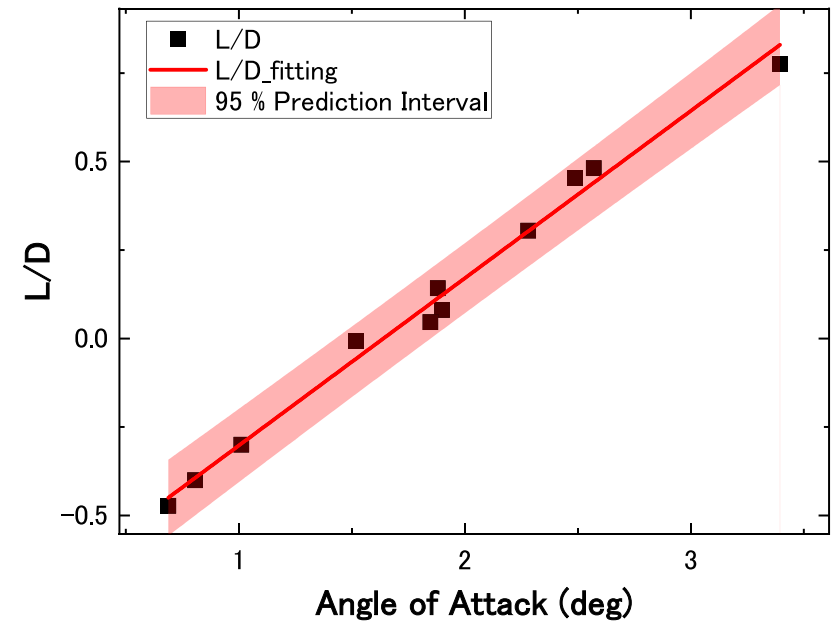

Fig. 13 The relationship between the model angle of attack and the lift-drag ratio

Table 5 The standard deviation of the current measurement

\begin{tabular}{ll}
\hline$C_{D}$ & 0.0073 \\
\hline$C_{L}$ & 0.011 \\
$C_{M}$ & 0.0021 \\
$L / D$ & 0.052 \\
\hline
\end{tabular}

$C_{M}=\frac{M_{y}}{\frac{1}{2} \rho_{\infty} U_{\infty}^{2} S L}$

The representative length $L$ is the total length of the model in the longitudinal direction. The representative area $S$ is that of the front cross section, which was calculated from CAD data. An example of the traces of the three-component aerodynamic coefficients is shown in Fig. 11. The traces were filtered with a Fourier filter with a cutoff frequency of $367 \mathrm{~Hz}$ (time constant $2.7 \mathrm{~ms}$ ). Low-frequency oscillation remains in the pitching moment trace, which may result from the model's primary bending mode. However, the coefficients appear constant throughout the test time.

Averaging the traces of the coefficients over the defined test time (2-6 ms), eleven data sets were obtained. These data sets are plotted in Figs. 12 and 13 to show the relationship between the angle of attack and aerodynamic coefficients and lift-drag ratio. A 95\% prediction interval band is overlapped as a hatch area on each coefficient line to show each measurement's precision. The standard deviation of all the aerodynamic coefficients at the present data set (degrees of freedom was ten) is summarized in Table 5.

In Fig. 12 (a, b), drag and lift coefficients show positive linear relationships with the angle of attack from 0.7 to 3.4 degrees. In Fig. 12(c), $C_{M}$ also increased in line with the angle of attack, making the model aerodynamically unstable. However, this is particular to the current model, in which the center of gravity is shifted downstream due to the batteries' location in the aft of the model. 


\section{Conclusion}

In this study, the three-component aerodynamic coefficients (Drag, Lift, and Pitching moment) of an airframe-engine integrated scramjet vehicle model MoDKI were successfully obtained in the free-piston shock tunnel HIEST. A free-flight force measurement technique was also adopted. A new analytical method was developed for multiple single-axis piezoelectric accelerometers based on an overdetermined system analysis. The current approach itself is not novel because it is based on general linear regression theory. However, a unique assumption, which is satisfied under short measurement time, heavy model, and differential-type (piezoelectric type) accelerometers, successfully simplified the dynamic equation of motion. The method allows multi-component forces and moments to be measured with a straightforward calculation using several single-axis accelerometers. It improves uncertainties originating from the measurement variability of each acceleration. Moreover, it also mitigates the troublesome issues for positioning accelerometers and may circumvent interference with the model's natural vibrations. Despite the relatively limited applicable scope (shortduration tests), the method should help free-flight force measurement in impulsive facilities.

Open Access This article is licensed under a Creative Commons Attribution 4.0 International License, which permits use, sharing, adaptation, distribution and reproduction in any medium or format, as long as you give appropriate credit to the original author(s) and the source, provide a link to the Creative Commons licence, and indicate if changes were made. The images or other third party material in this article are included in the article's Creative Commons licence, unless indicated otherwise in a credit line to the material. If material is not included in the article's Creative Commons licence and your intended use is not permitted by statutory regulation or exceeds the permitted use, you will need to obtain permission directly from the copyright holder. To view a copy of this licence, visit http://creativecommons.org/licenses/by/4.0/.

\section{References}

Bernstein L (1975) Force measurement in short-duration hypersonic facilities. AGARD-AG-214, edited by R. C. Pankhurst (Technical Editing and Reproduction, London).

Doherty LJ, Smart MK, Mee DJ (2015) Measurement of three-components of force on an airframe integrated scramjet at Mach 10. In 20th AIAA International Space Planes and Hypersonic Systems and Technologies Conference (p. 3523). https://doi.org/10.2514/6. 2015-3523

Duryea GR, Sheeran WJ (1969) Accelerometer force balance techniques. International Congress on Instrumentation in Aerospace Simulation Facilities'69 Record, 190-197.

Hannemann K, Schramm JM, Laurence SJ, Karl S, Langener T, Steelant J (2014) Experimental and numerical analysis of the small scale LAPCAT II scramjet flow path in high enthalpy shock tunnel conditions. Space Propulsion, May 19-22 2014, Cologne, Germany, SP2014-2969350.

Hannemann K, Schramm JM, Karl S, Laurence SJ (2015) Free flight testing of a scramjet engine in a large scale shock tunnel. 20th
AIAA International Space Planes and Hypersonic Systems and Technologies Conference, AIAA Paper 2015-3608. https://doi. org/10.2514/6.2015-3608

Holden M (2000) Studies of scramjet performance in the LENS facilities, 36th AIAA/ASME/SAE/ASEE Joint Propulsion Conference July 17-19, 2000, Huntsville, AIAA Paper No. 2000-3604. https:// doi.org/10.2514/6.2000-3604

Itoh K, Ueda S, Komuro T, Sato K, Takahashi M, Miyajima H, Tanno $\mathrm{H}$, Muramoto $\mathrm{H}$ (1998) Improvement of a free piston driver for a high-enthalpy shock tunnel. Shock Waves 8:215-233. https://doi. org/10.1007/s001930050115

Itoh K, Ueda S, Tanno H, Komuro T, Sato K (2002) Hypersonic aerothermodynamic and scramjet research using high enthalpy shock tunnel. Shock Waves 12:93-98. https://doi.org/10.1007/ s00193-002-0147-0

Jiang Z, Zhan Z, Liu Y, Wang C (2021) Luo C (2021) Criteria for hypersonic airbreathing propulsion and its experimental verification. Chin J Aeronaut 34(3):94-104. https://doi.org/10.1016/j.cja. 2020.11.001

Joshi MV, Reddy NM (1986) Aerodynamic force measurements over missile configurations in IISc shock tunnel at $\mathrm{M}_{\infty}=5.5$. Exp Fluids. https://doi.org/10.1007/BF00266300

Laurence SJ (2012) On tracking the motion of rigid bodies through edge detection and least-squares fitting. Exp Fluids 52(2):387401. https://doi.org/10.1007/s00348-011-1228-6

Naumann KW, Ende H, Mathieu G, George A (1993) Millisecond aerodynamic force measurement with side-jet model in the ISL shock tunnel. AIAA J 31(6):1068-1074. https://doi.org/10.2514/3.11730

Padgaonkar AJ, Krieger KW, King AI (1975) Measurement of angular acceleration of a rigid body using linear accelerometers. J Appl Mech 42(3):552-554. https://doi.org/10.1115/1.3423640

Paull A, Stalker RJ, Mee DJ (1995) Experiments on supersonic combustion ramjet propulsion in a shock tunnel. J Fluid Mech. https:// doi.org/10.1017/S0022112095002096

Reddy NM (1983) Aerodynamic force measurements in IISc hypersonic shock tunnel. Proc. of XIV International symposium on shock tubes and shock waves, Sydney, Australia, 358-362.

Robinson MJ, Mee DJ, Tsai CY, Bakos RJ (2004) Three-component force measurements on a large scramjet in a shock tunnel. J Spacecr Rocket 41(3):416-425

Robinson MJ, Mee DJ, Paull A (2006) Scramjet lift, thrust and pitching-moment characteristics measured in a shock tunnel. J Propul Power 22(1):85-95. https://doi.org/10.2514/1.15978

Stalker RJ, Morgan RG (1982) Parallel hydrogen injection into constant-area, high-enthalpy, supersonic airflow. AIAA J 20(10):1468-1469

Takahashi M, Kodera M, Itoh K, Komuro T, Sato K, Tanno H (2009) Influence of thermal non-equilibrium on nozzle flow condition of high enthalpy shock tunnel HIEST. 16th AIAA/DLR/DGLR International Space Planes and Hypersonic Systems and Technologies Conference, AIAA Paper 2009-7267. https://doi.org/10. 2514/6.2009-7267

Tanno H, Kodera M, Komuro T, Sato K, Itoh K, Takahashi M (2009) Experimental and numerical studies to evaluate real-gas effects on generic models in the free-piston shock tunnel HIEST. Proceedings of the 6th European Symposium on Aerothermodynamics for Space Vehicles, by Ouwehand, L. Noordwijk, Netherlands: European Space Agency, 2009, id.120.

Tanno H, Komuro T, Sato K, Fujita K, Laurence SJ (2014) Free-flight measurement technique in the free-piston high-enthalpy shock tunnel. Rev Sci Instrum. https://doi.org/10.1063/1.4870920

Publisher's note Springer Nature remains neutral with regard to jurisdictional claims in published maps and institutional affiliations. 